\title{
Complexities and protein complexes in the antimycin A-sensitive pathway of cyclic electron flow in plants
}

\author{
Dario Leister $^{1,2 *}$ and Toshiharu Shikanai ${ }^{3}$ \\ Department Biology I, Plant Molecular Biology (Botany), Ludwig-Maximilians-University Munich, Munich, Germany \\ 2 PhotoLab Trentino - A Joint Initiative of the University of Trento (Centre for Integrative Biology) and the Edmund Mach Foundation \\ (Research and Innovation Centre), San Michele all'Adige and Mattarello, Italy \\ ${ }^{3}$ Department of Botany, Graduate School of Science, Kyoto University, Kyoto, Japan \\ *Correspondence: leister@Imu.de
}

Edited by:

Helmut Kirchhoff, Washington State University, USA

Reviewed by:

Helmut Kirchhoff, Washington State University, USA

Antimycin A-sensitive cyclic electron flow (AA-sensitive CEF) was discovered by Arnon and co-workers more than 50 years ago and serves to recycle electrons from ferredoxin $(F d)$ to plastoquinone $(P Q)$. A role in $A A-$ sensitive CEF has been attributed to the two thylakoid proteins PGR5 and PGRL1 ever since their identification, but this assignment remains controversial. While current technical limitations have prevented unequivocal clarification of their precise function in CEF in vivo, recent biochemical experiments have implied that PGRL1/PGR5 complexes possess Fd$P Q$ reductase $(F Q R)$ activity in vitro. Consequently, PGRL1-PGR5 complexes in flowering plants appear to shuttle between photosystem I (PSI) and the cytochrome (Cyt) $b_{6} f$ complex, whereas in the green alga Chlamydomonas PGRL1 (but not PGR5) has been detected in a PSI-Cyt $b_{6} f$ supercomplex that has intrinsic CEF activity.

\section{A BRIEF LOOK BACK}

Cyclic electron flow (CEF) around photosystem I (PSI) recycles electrons from $\mathrm{Fd}$ to $P Q$, generating ATP without accumulation of NADPH (reviewed in: Shikanai, 2007) (see Figure 1A). The discovery of CEF dates back to 1954, when Arnon and co-workers identified, in isolated chloroplasts, a process that generates ATP without production of $\mathrm{O}_{2}$ (Arnon et al., 1954), requires $\mathrm{Fd}$ and is sensitive to antimycin A (AA) (Tagawa et al., 1963). The Arnon group then extended their work to the identification of another type of electron flow, namely non-cyclic (or linear) electron flow (LEF) (Arnon et al., 1958), which is now widely accepted as the major pathway for the light reactions of photosynthesis. Today, investigation of the function of the LEF machinery has reached the atomic level (Umena et al., 2011), but the molecular details and physiological significance of CEF remain largely elusive- a situation which is largely due to technical constraints on our ability to measure CEF directly, especially in leaves (reviewed in: Johnson, 2005). Indeed, while the scepticism encountering the concept of ATP production in chloroplasts has disappeared over the years (Arnon, 1991), doubts about the validity of the concept of CEF persist.

\section{THE GENETIC EVIDENCE}

A breakthrough in CEF research was achieved with the discovery that a multiprotein complex, which resembles Complex I in the mitochondrial respiratory chain, mediates CEF in cyanobacteria (reviewed in: Ogawa and Mi, 2007). This made the equivalent complex in chloroplasts, the so-called "NAD $(\mathrm{P}) \mathrm{H}$ dehydrogenase" or NDH complex, a prime candidate for a component of CEF in algae and plants. Analysis of knockout mutants of plastid $n d h$ genes in tobacco then showed that chloroplast NDH indeed mediates electron transport from stromal reductants to $\mathrm{PQ}$, although photosynthesis is not overtly affected in these mutants, at least under greenhouse conditions (Burrows et al., 1998; Shikanai et al., 1998). Chloroplast NDH most probably accepts electrons from $\mathrm{Fd}$ rather than NAD(P)H (Yamamoto et al., 2011), but it is insensitive to AA (Endo et al., 1997). Consequently, Arnon's CEF cannot be mediated by the NDH complex and, to avoid confusion, we will refer to the process originally described by Arnon as "AA-sensitive CEF."

AA-sensitive CEF was finally "rediscovered" in the course of a screen for Arabidopsis mutants defective in the induction of non-photochemical quenching (NPQ) at high light intensity (Munekage et al., 2002). In the mutant proton gradient regulation 5 ( $p g r 5$ ), the PSI reaction Centre $\left(\mathrm{P}_{700}\right)$ is highly reduced in the light, whereas it is oxidized in the wild type (Munekage et al., 2002). This $\mathrm{P}_{700}$ phenotype is probably due to decreased production of ATP, which leads to photoinhibition of PSI. Soon after that report, the Arabidopsis chlororespiratory reduction (crr) mutants, which are defective in $\mathrm{NDH}$ activity, were isolated in a screen based on chlorophyll fluorescence imaging (Hashimoto et al., 2003). Although both pgr5 and $\mathrm{cr}$ single mutants behave like wild type under standard conditions, growth and photosynthesis are severely impaired in $\mathrm{crr}$ pgr5 double mutants (Munekage et al., 2004).

To demonstrate that the pgr5 and $\mathrm{crr}$ mutants are indeed defective in CEF, an assay was employed in which $\mathrm{Fd}$ dependent PQ reduction activity was measured in ruptured chloroplasts. With this assay, clear and additive effects on chlorophyll fluorescence were observed for the crr and pgr5 mutants, and were ascribed to lesions in the NDH- and AA-sensitive pathways of CEF, respectively (Munekage et al., 2002, 2004). However, these conclusions are based on phenotypical analyses of the relative severity of the CEF lesion in the mutants concerned, and are thus constrained by the methodological 


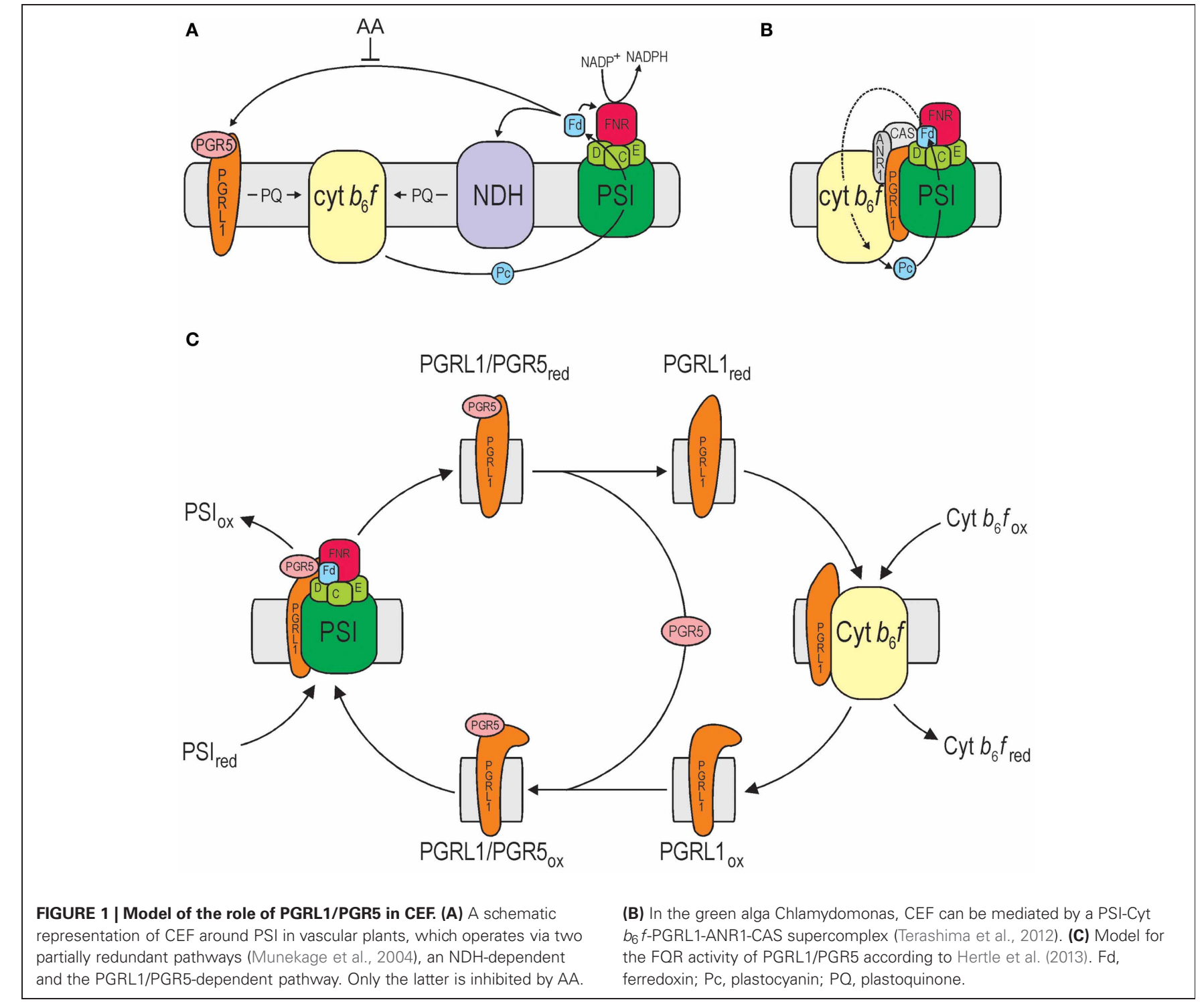

B

limitations mentioned above. Hence, in the absence of clear phenotypic criteria for deciding when a photosynthetic mutant qualifies as a true AA-CEF mutant, an alternative and more indirect role for PGR5 [and its interacting partner PGRL1 (DalCorso et al., 2008)] in CEF has been proposed (see: Nandha et al., 2007).

However, it is worth pointing out here that, irrespective of the status of the CEF measurements, the following major conclusions derived from the analyses of the pgr5 mutant remain valid: (1) PGR5 and PGRL1 are necessary for NPQ induction and protection of PSI from photoinhibition (Munekage et al., 2002; DalCorso et al., 2008). (2) The pgr5 mutant is sensitive to fluctuating light levels (Suorsa et al., 2012) and the crr pgr5 double mutant is sensitive even to constant low light (Munekage et al., 2004). (3) In the ruptured chloroplast assay, AA affects chlorophyll fluorescence in the WT but not in pgr5 plants (Munekage et al., 2002, 2004). Taken together, both the role of PGR5 in photoprotection $(1+2)$ and its relationship to sensitivity to AA (3) provide a reasonable basis for the hypothesis that PGR5 is involved in AA-sensitive CEF.

\section{BIOCHEMICAL ANALYSES}

The transmembrane protein PGRL1 was identified in thylakoids of Arabidopsis by DalCorso et al. (2008). Because plants lacking PGRL1 showed a perturbation of CEF similar to that seen in PGR5-deficient plants, the protein was named "PGR5like protein 1" or PGRL1. Yeast twohybrid and split-ubiquitin assays, as well as PSI co-purification experiments demonstrated that PGRL1 and PGR5 interact with each other and with PSI. PGRL1 also interacts with $\mathrm{Fd}$, the Fd-NADPH oxidoreductase (FNR) and the cytochrome (Cyt) $b_{6} f$ subunit Cyt $b_{6}$-at least in yeast assays. Moreover, mutants lacking PGRL1 do not accumulate PGR5, whereas PGR5less plants still express PGRL1, suggesting that PGRL1 represents the docking site for PGR5. Based on these data, it was proposed that the PGRL1-PGR5 complex, together with Fd and the FNR, represents the long-sought Fd:PQ reductase (FQR) and facilitates AA-sensitive CEF in 
plants, while spectroscopic data were also adduced in support of a regulatory role for the complex (DalCorso et al., 2008). In the green alga Chlamydomonas, PGRL1 functions in iron sensing (Petroutsos et al., 2009) in addition to regulating CEF in conjunction with ANR1 and CAS, and all three are associated with each other in a multiprotein complex (Terashima et al., 2012; see Figure 1B).

In a more recent study, PGRL1 was found to be a redox-active protein that exists in monomeric and homodimeric forms, and as a heterodimer with PGR5 (Hertle et al., 2013) (see Figure 1C). Moreover, co-immunoprecipitation assays confirmed the interaction of PGRL1 with Cyt $b_{6}$. Systematic mutagenesis of six conserved cysteine residues in PGRL1 identified three functional redox-regulated domains: a homodimerization motif that is regulated by thioredoxins, an ironcontaining cofactor site and a domain required for heterodimerization with PGR5. In-vitro assays showed that the PGRL1-PGR5 complex can accept electrons from Fd, whereas PGRL1 alone is sufficient to reduce quinones. In addition, the redox kinetics of PGRL1 in planta confirm that it receives electrons from PSI in a PGR5-dependent manner. Taken together, these results strongly argue that PGRL1 acts as the plant FQR, with PGR5 playing an accessory but essential role (Hertle et al., 2013).

\section{INTERSPECIFIC DIVERSITY}

The function of the PGRL1/PGR5dependent CEF pathway seems to be conserved in flowering plants, as indicated by the phenotype of PGR5 knock-down lines of rice, which in many respects resembles that of Arabidopsis pgr5 mutants (Nishikawa et al., 2012). In contrast, cyanobacteria appear to lack the PGRL1 protein. However, inactivation of a cyanobacterial gene that displays weak homology to PGR5 appears to perturb cyanobacterial AA-sensitive CEF (Yeremenko et al., 2005). Homologues of both PGR5 and PGRL1 exist in the green alga Chlamydomonas reinhardtii. However, in contrast to the situation in land plants (where a PSI-Cyt $b_{6} f$ supercomplex has not been detected yet), in Chlamydomonas PGRL1 is part of a supercomplex that contains PSI and the Cyt $b_{6} f$ complex (Iwai et al., 2010) (see Figure 1B), together with the algal-specific protein ANR1 and the protein CAS, which is conserved in algae and vascular plants (Terashima et al., 2012). Moreover, (1) Chlamydomonas CEF appears not to be sensitive to AA (Iwai et al., 2010), and (2) as in other species of Chlorophyceae, $n d h$ genes are absent in Chlamydomonas. Interestingly, the Chlamydomonas PGR5 protein has yet to be detected in the PSI-Cyt $b_{6} f$-PGRL1-ANR1-CAS supercomplex.

One may therefore speculate that PGRL1/PGR5-dependent CEF evolved from a process that requires PGR5 only (Synechocystis) to one that entails cooperation between the two proteins-in the context of either an AA-insensitive supercomplex (Chlamydomonas) or an AA-sensitive heterodimer (Arabidopsis).

\section{CONTROVERSIES}

The role of PGR5/PGRL1 in CEF is still very much a live issue. We will now review the arguments advanced against the involvement of PGR5/PGRL1 in AA-sensitive CEF.

1. Doubts have been raised regarding the reliability of the ruptured chloroplast assay (see above) as an indicator of CEF rates in vivo (Nandha et al., 2007). The kinetics of chlorophyll fluorescence responses observed in this assay are certainly too slow to reflect in-vivo rates of CEF directly. But this feature, which can plausibly be attributed to functional impairment of ruptured relative to intact chloroplasts, need not necessarily exclude the possibility that the assay provides an indirect measure of CEF. In fact, the additivity of the effects of $\mathrm{cr} r$ and pgr 5 mutations, together with the impact of AA, on chlorophyll fluorescence kinetics indicate that this assay, at least qualitatively, captures a process that is closely linked to CEF.

2. Results from $\mathrm{P}_{700}$ oxidation kinetics and certain chlorophyll fluorescence measurements done on leaves have been taken as evidence against a direct involvement of PGR5 in CEF (Nandha et al., 2007). However, these techniques also do not measure $\mathrm{CEF}$ directly, and some of them fail to detect an inhibitory effect of AA on CEF (Joliot and Joliot, 2002).

3. Avenson et al. (2005) monitored electrochromic shift (ECS) signals and found that the absence of PGR5 affects a flux of protons corresponding to $\sim 13 \%$ of that from LEF. This finding can be explained in two ways. Either AA-sensitive CEF contributes around $13 \%$ to the total proton motive force $(p m f)$ (Avenson et al., 2005), or the total contribution of CEF is markedly higher than $13 \%$ and CEF is only partially suppressed in pgr5 mutants, which would point to a regulatory role of PGR5/PGRL1 in CEF (Nandha et al., 2007). Here the same objection can be raised as before: like the ruptured chloroplast or $\mathrm{P}_{700}$ kinetics assays discussed above, the ECS approach itself does not directly monitor the rate of CEF but the pmf, which is the primary product of CEF. Puzzlingly, proton conductivity $\left(\mathrm{g}_{\mathrm{H}^{+}}\right)$is increased in Arabidopsis pgr5 (Avenson et al., 2005) and rice PGR5 knockdown lines (Nishikawa et al., 2012). It seems unlikely that PGR5 directly regulates the ATPase, but perhaps ATP production is somehow upregulated to compensate for the lack of CEF in pgr5 plants (Nishikawa et al., 2012).

4. Suorsa et al. (2012) have suggested that PGR5 protects PSI from photoinhibition by regulating LEF, in particular Cyt $b_{6} f$ activity. This idea actually does not conflict with the concept that PGRL1/PGR5 mediates CEF. A tentative 13\% contribution of PGRL1/PGR5-dependent CEF to total pmf is not insignificant and the direct CEF-related effect of the pgr5 mutation on lumen $\mathrm{pH}$ might be exacerbated by the altered regulation of proton conductivity (see above). In consequence, PGR5 could regulate Cyt $b_{6} f$ indirectly via its primary effect on lumen acidification during CEF.

\section{CONCLUSIONS}

We currently lack a reliable way of measuring CEF directly, and thus have no means of clearly distinguishing between partial and complete lack of CEF. Conversely, without mutants that are generally 
acknowledged to lack CEF specifically, it is impossible to establish and calibrate methods that are suitable for quantifying CEF. Therefore, a crucial step in elucidating AA-sensitive CEF will be to corroborate the function of PGRL1 and PGR5 as an FQR and address the basis for the apparently different functions of PGRL1 and PGR5 in plants and green algae.

\section{ACKNOWLEDGMENTS}

We thank Paul Hardy for critical comments on the manuscript.

\section{REFERENCES}

Arnon, D. I. (1991). Photosynthetic electron transport: emergence of a concept, 1949-1959. Photosynth. Res. 29, 117-131.

Arnon, D. I., Allen, M. B., and Whatley, F. R. (1954). Photosynthesis by isolated chloroplasts. Nature 174, 394-396.

Arnon, D. I., Whatley, F. R., and Allen, M. B. (1958). Assimilatory power in photosynthesis: photosynthetic phosphorylation by isolated chloroplasts is coupled with TPN reduction. Science 127, 1026-1034.

Avenson, T. J., Cruz, J. A., Kanazawa, A., and Kramer, D. M. (2005). Regulating the proton budget of higher plant photosynthesis. Proc. Natl. Acad. Sci. U.S.A. 102, 9709-9713.

Burrows, P. A., Sazanov, L. A., Svab, Z., Maliga, P., and Nixon, P. J. (1998). Identification of a functional respiratory complex in chloroplasts through analysis of tobacco mutants containing disrupted plastid ndh genes. EMBO J. 17, 868-876.

DalCorso, G., Pesaresi, P., Masiero, S., Aseeva, E., Schünemann, D., Finazzi, G., et al. (2008). A complex containing PGRL1 and PGR5 is involved in the switch between linear and cyclic electron flow in Arabidopsis. Cell 132, 273-285.

Endo, T., Mi, H., Shikanai, T., and Asada, K. (1997). Donation of electrons to plastoquinone by $\mathrm{NAD}(\mathrm{P}) \mathrm{H}$ dehydrogenase and by ferredoxin-quinone reductase in spinach chloroplasts. Plant Cell Physiol. 38, 1272-1277.
Hashimoto, M., Endo, T., Peltier, G., Tasaka, M., and Shikanai, T. (2003). A nucleus-encoded factor, CRR2, is essential for the expression of chloroplast $n d h B$ in Arabidopsis. Plant J. 36, 541-549.

Hertle, A. P., Blunder, T., Wunder, T., Pesaresi, P., Pribil, M., Armbruster, U., et al. (2013). PGRL1 is the elusive ferredoxin-plastoquinone reductase in photosynthetic cyclic electron flow. Mol. Cell 49, 511-523.

Iwai, M., Takizawa, K., Tokutsu, R., Okamuro, A. Takahashi, Y., and Minagawa, J. (2010). Isolation of the elusive supercomplex that drives cyclic electron flow in photosynthesis. Nature 464, 1210-1213.

Johnson, G. N. (2005). Cyclic electron transport in $\mathrm{C}_{3}$ plants: fact or artefact? J. Exp. Bot. 56, 407-416.

Joliot, P., and Joliot, A. (2002). Cyclic electron transfer in plant leaf. Proc. Natl. Acad. Sci. U.S.A. 99, 10209-10214.

Munekage, Y., Hashimoto, M., Miyake, C., Tomizawa, K., Endo, T., Tasaka, M., et al. (2004). Cyclic electron flow around photosystem I is essential for photosynthesis. Nature 429, 579-582.

Munekage, Y., Hojo, M., Meurer, J., Endo, T., Tasaka, M., and Shikanai, T. (2002). PGR5 is involved in cyclic electron flow around photosystem I and is essential for photoprotection in Arabidopsis. Cell $110,361-371$

Nandha, B., Finazzi, G., Joliot, P., Hald, S., and Johnson, G. N. (2007). The role of PGR5 in the redox poising of photosynthetic electron transport. Biochim. Biophys. Acta 1767, 1252-1259.

Nishikawa, Y., Yamamoto, H., Okegawa, Y., Wada, S., Sato, N., Taira, Y., et al. (2012). PGR5-dependent cyclic electron transport around PSI contributes to the redox homeostasis in chloroplasts rather than $\mathrm{CO}_{2}$ fixation and biomass production in rice. Plant Cell Physiol. 53, 2117-2126.

Ogawa, T., and Mi, H. (2007). Cyanobacterial NADPH dehydrogenase complexes. Photosynth. Res. 93, 69-77.

Petroutsos, D., Terauchi, A. M., Busch, A., Hirschmann, I., Merchant, S. S., Finazzi, G., et al. (2009). PGRL1 participates in iron-induced remodeling of the photosynthetic apparatus and in energy metabolism in Chlamydomonas reinhardtii. J. Biol. Chem. 284, 32770-32781.

Shikanai, T. (2007). Cyclic electron transport around photosystem I; genetic approaches. Annu. Rev. Plant Biol. 58, 199-217.
Shikanai, T., Endo, T., Hashimoto, T., Yamada, Y., Asada, K., and Yokota, A. (1998). Directed disruption of the tobacco $n d h B$ gene impairs cyclic electron flow around photosystem I. Proc. Natl. Acad. Sci. U.S.A. 95, 9705-9709.

Suorsa, M., Järvi, S., Grieco, M., Nurmi, M., Pietrzykowska, M., Rantala, M., et al. (2012). PROTON GRADIENT REGULATION5 is essential for proper acclimation of Arabidopsis photosystem I to naturally and artificially fluctuating light conditions. Plant Cell 24, 2934-2948.

Tagawa, K., Tsujimoto, H. Y., and Arnon, D. I. (1963). Role of chloroplast ferredoxin in the energy conversion process of photosynthesis. Proc. Natl. Acad. Sci. U.S.A. 49, 567-572.

Terashima, M., Petroutsos, D., Hüdig, M., Tolstygina, I., Trompelt, K., Gäbelein, P., et al. (2012). Calcium-dependent regulation of cyclic photosynthetic electron transfer by a CAS, ANR1, and PGRL1 complex. Proc. Natl. Acad. Sci. U.S.A. 109, 17717-17722.

Umena, Y., Kawakami, K., Shen, J. R., and Kamiya, N. (2011). Crystal structure of oxygen-evolving photosystem II at a resolution of $1.9 \AA$. Nature 473 , 55-60.

Yamamoto, H., Peng, L., Fukao, Y., and Shikanai, T. (2011). An Src homology 3 domain-like fold protein forms a ferredoxin-binding site for the chloroplast NADH dehydrogenase-like complex in Arabidopsis. Plant Cell 23, 1480-1493.

Yeremenko, N., Jeanjean, R., Prommeenate, P., Krasikov, V., Nixon, P. J., Vermaas, W. F., et al. (2005). Open reading frame ssr2016 is required for antimycin A-sensitive photosystem I-driven cyclic electron flow in the cyanobacterium Synechocystis sp. PCC (6803). Plant Cell Physiol. 46, 1433-1436.

Received: 24 April 2013; accepted: 09 May 2013; published online: 27 May 2013.

Citation: Leister D and Shikanai T (2013) Complexities and protein complexes in the antimycin A-sensitive pathway of cyclic electron flow in plants. Front. Plant Sci. 4:161. doi: 10.3389/fpls.2013.00161

This article was submitted to Frontiers in Plant Physiology, a specialty of Frontiers in Plant Science.

Copyright (c) 2013 Leister and Shikanai. This is an open-access article distributed under the terms of the Creative Commons Attribution License, which permits use, distribution and reproduction in other forums, provided the original authors and source are credited and subject to any copyright notices concerning any thirdparty graphics etc. 